\title{
Magnetic Fine Particles in Biological Systems
}

\author{
Dominic P.E. Dickson ${ }^{a}$ and Richard B. Frankel ${ }^{b}$

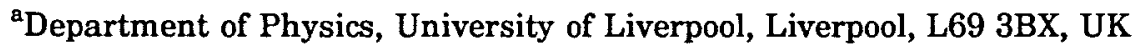 \\ bPhysics Department, California Polytechnic State University, San Luis Obispo, \\ California, CA93407, USA
}

\begin{abstract}
Two examples of biological magnetic fine particles are considered: ironstorage proteins and magnetotactic bacteria. These cover many aspects of this field, including the use of well-defined biological systems for testing theoretical models, using magnetic properties to distinguish between different biological materials, producing magnetic materials by biological processes, and using optimised biological magnetic systems as a guide to the production of synthetic magnetic materials.
\end{abstract}

\section{INTRODUCTION}

Biological systems provide a number of examples of magnetic fine particles in which a variety of different areas of interest can be identified.

One aspect of this field is the way in which some of the biological materials provide well-defined model systems which can be used to test theoretical ideas concerning fine particle magnetism. The naturally occurring materials can be modified by in vitro processes following extraction, by altering growth conditions, and even genetically. In this way it may be possible to produce technologically important magnetic materials using biologically related procedures.

In many cases the magnetic properties are essentially coincidental to the primary role of the biological material. However, these magnetic properties do provide a convenient and important diagnostic for understanding the structure and properties of the material and for identifying crucial differences between related materials.

In a number of cases the magnetic properties are the primary and indeed only role of the material. In these situations it appears that the living organism has evolved methods of producing magnetic fine particles which optimise their magnetic effectiveness for direction-finding.

The biological systems can also be used as a guide to a strategy for the design of novel materials and structures. The rationale for this "biomimicking" is that the materials found in living organisms have been refined and optimised 
in the course of evolution, over a very long timescale, to have certain properties and functions.

Two examples have been chosen to illustrate these various aspects of magnetic fine particles in biological systems: iron-storage proteins and magnetotactic bacteria.

\section{IRON-STORAGE PROTEINS}

\subsection{Ferritin and Haemosiderin}

Almost all living organisms require the element iron for essential metabolic functions. The iron is found in a wide range of biomolecules with many different roles. A good example is haemoglobin, the oxygen transport protein found in all higher animals. The importance of iron leads to the necessity for a source of iron, from which the iron-containing proteins can be synthesized. In addition, because of the chemical reactivity and hence toxicity of free iron, some form of encapsulation is required, to render inert any stored or excess iron which may be present. These functions are carried out by the iron-storage proteins ferritin and haemosiderin.

Ferritin and haemosiderin contain an inorganic mineral iron-containing core surrounded by protein. In the case of ferritin the protein forms a complete spherical shell with an external diameter of $12 \mathrm{~nm}$ and an internal diameter of approximately $8 \mathrm{~nm}[1,2]$. Thus ferritin has a well-defined cavity in which the iron-containing mineral core is formed. This cavity can be filled with up to about 4000 iron atoms in the form of the iron oxyhydroxide mineral ferrihydrite.

Haemosiderin, like ferritin, has a mineral iron core, but with only a partial protein envelope, rather than a complete shell [3]. The haemosiderin core is not generally as well crystallized and does not have as well defined a shape as is the case with the ferritin core, and in addition, the mineral form of the core appears to be variable [4].

\subsection{Superparamagnetism}

Bulk ferrihydrite becomes magnetically ordered at temperatures between 100 and $200 \mathrm{~K}$. However, the core sizes found in samples of ferritin and haemosiderin are such that superparamagnetic relaxation, whereby the magnetization vector undergoes thermally driven reversals, occurs below the ordering temperature. The relatively narrow particle size distribution, together with the separation of the magnetic particles resulting from the protein shell, make ferritin an ideal model system for investigating this phenomenon. Mössbauer spectroscopy provides an appropriate technique for the observation of the superparamagnetic behaviour [5]. 
For uniaxial anisotropy, the timescale for magnetization reversal can be expressed by the equation proposed by Néel [6]:

$\tau=\tau_{0} \exp \left(E_{\mathrm{A}} / k T\right)$

The pre-exponential factor $\tau_{0}$ is usually considered to be a constant, characteristic of the material, although more complex forms for this factor have also been proposed [7]. The anisotropy energy of a particle $E_{\mathrm{A}}$ depends on many factors and in a particular sample there will be a distribution $p\left(E_{\mathrm{A}}\right)$. Thus at a particular temperature there will be a distribution of relaxation times $p(\tau)$. The observed Mössbauer spectrum at this temperature will be the sum of the spectra corresponding to a combination of the hyperfine parameters of the material and the various values of $\tau$ within the distribution. Computer fitting a series of Mössbauer spectra at different temperatures can in principle yield both $\tau_{0}$ and $p\left(E_{\mathrm{A}}\right)$ for the sample. Such a set of fits to the superparamagnetic Mössbauer spectra of ferritin are shown in Figure 1 [8].

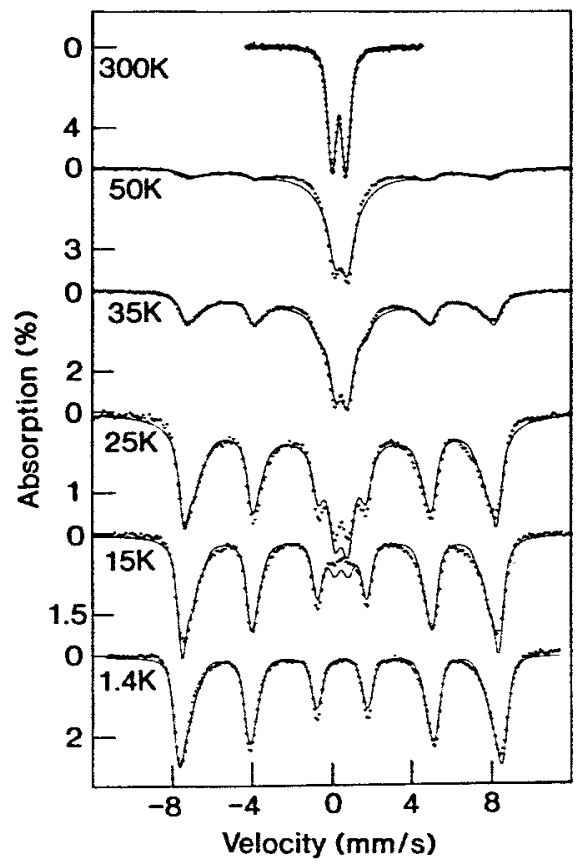

Figure 1 Variable temperature Mössbauer spectra of horse spleen ferritin. The line through the experimental points is the computer fit to the superparamagnetic relaxation model discussed in the text. 
The model provides good fits to the experimental data, although the interrelationship between $\tau_{\mathrm{o}}$ and $p\left(E_{\mathrm{A}}\right)$ make it unlikely that unique values of these parameters can be obtained by Mössbauer spectroscopy alone. However, a combination of Mössbauer spectroscopy and bulk magnetic measurements on the same sample should allow these parameters to be determined. Such an investigation will also provide valuable information on the validity of the Neel equation as a description of the superparamagnetic behaviour of real systems.

The biological control of the magnetic small particles in ferritin, and hence the well-defined nature of the particles, make this iron-storage protein a particularly appropriate system for an investigation of this type. Modification of the distribution of particle sizes within the sample by various biochemical procedures during extraction may be additionally helpful [9].

\subsection{Iron-Overload Diseases}

Certain diseases lead to the build up of iron in the body. These diseases fall into two main groups. In primary haemochromatosis the iron overload is a consequence of a breakdown of a "switch" in the gut which controls the uptake of iron. In secondary haemochromatosis the excess iron results from multiple blood transfusions administered because of a genetic blood disease. Certain animals also exhibit iron overload. In all these cases the iron is predominantly in the form of insoluble granules with associated protein, known as haemosiderin [10-12]. Determining the form of the iron in the haemosiderin should considerably advance our understanding of the nature of these diseases and help in devising appropriate treatment strategies.

Striking differences in magnetic behaviour of these haemosiderins, observed by means of Mössbauer spectroscopy, have enabled three distinct forms of haemosiderin to be clearly identified [4,13]. In iron-overloaded animals, normal humans and untreated primary haemochromatosis, the haemosiderin (Hd1) shows superparamagnetic temperature dependent behaviour very similar to that of ferritin. In the case of secondary haemochromatosis haemosiderin (Hd2), superparamagnetic behaviour is also observed but with magnetic splitting persisting at much higher temperatures, indicating a material with much greater magnetic anisotropy. The haemosiderin from treated primary haemochromatosis (Hd3) shows quite different Mössbauer spectra, with a magnetic ordering transition at very low temperatures, indicative of a material with much smaller magnetic exchange.

This clear evidence from the magnetic behaviour, for three different forms of haemosiderin, has subsequently been confirmed by other techniques, and in particular electron diffraction [4]. The electron diffraction data show that Hd1 has iron-containing cores consisting of ferrihydrite, as in the case of ferritin, while in $\mathrm{Hd} 2$ the cores are goethite-like, and in $\mathrm{Hd} 3$ the cores are amorphous. This information has important consequences for understanding the nature of the iron overload in these relatively widespread diseases and for improving their treatment. 


\section{MAGNETOTACTIC BACTERIA}

\subsection{Magnetotaxis}

The process of magnetotaxis, by which certain aquatic bacteria orient and migrate along geomagnetic field lines, was originally found by accident $[14,15]$. Bacteria collected from marine and freshwater muds accumulate at the North side of drops of water and sediment on a microscope slide. They were found to swim towards the south pole and away from the north pole of a bar magnet. Experiments with Helmholtz coils showed that the bacteria from the Northern hemisphere swim along the field lines in the direction of the field, i.e. in the direction indicated by the North-seeking pole of a compass needle. These bacteria are therefore referred to as North-seeking. Reversals in the field direction lead the bacteria to do a "U-turn". Killed bacterial cells orient in the direction of the field but do not move along the field lines. Thus, motile magnetotactic bacteria behave like self-propelled permanent magnetic dipoles $[16,17]$.

The bacteria propel themselves forwards by means of a propeller-like appendage, the flagellum. Magnetotactic bacteria can have one of two magnetic polarities, depending on the orientation of a magnetic dipole contained within the cell [18]. Cells with the North-seeking pole forward with respect to the flagellum propel themselves parallel to the field direction (i.e. Northward), while those cells which have the South-seeking pole forward propel themselves antiparallel to the field direction (i.e. Southward). This behaviour is shown in Figure 2.

MAGNETIC FIELD

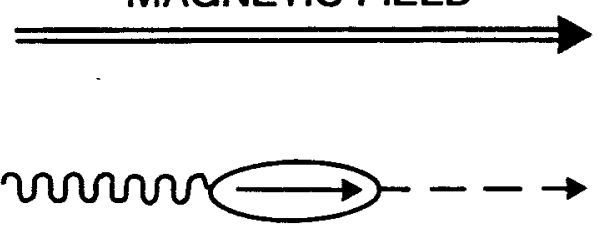

NORTH-SEEKING BACTERIUM

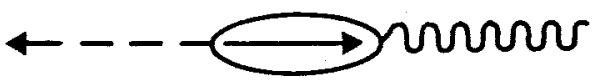

SOUTH-SEEKING BACTERIUM

Figure 2. Illustration of North-seeking and South-seeking bacteria in a magnetic field. 
North-seeking bacteria predominate in the Northern hemisphere, while in the Southern hemisphere the bacteria are predominantly South-seeking [19]. The vertical component of the inclined geomagnetic field selects the predominant polarity in each hemisphere by presumably favouring those cells whose polarity causes them to move downwards towards the sediment and away from the oxygenrich waters higher up which would be toxic to them. At the geomagnetic equator, where the vertical component of the magnetic field is zero, both North-seeking and South-seeking bacteria are found in equal numbers [20]. This is presumably because horizontally directed motion is advantageous in reducing upward motion that would be harmful. It has been found that an angle of inclination of only 6 to 8 degrees is sufficient to select one predominant polarity [21].

\subsection{The Magnetosome}

The permanent magnetic dipole moment of each magnetotactic cell is due to intracellular, membrane-bounded, permanent single-magnetic domain sized inorganic particles, known as magnetosomes, which are, in most cases, arranged in chains $[15,17,22,23]$. The biomineralization process, which involves the size, position, orientation, and even morphology of the particles, is highly controlled by the bacteria [24]. Moreover the magnetosome chain is a hierarchical structure that is a masterpiece of permanent magnet engineering.

The magnetosomes of most of the magnetotactic bacteria that have been studied to date contain particles of magnetite, $\mathrm{Fe}_{3} \mathrm{O}_{4}$, in the 40 to $100 \mathrm{~nm}$ size range. Recently, magnetotactic bacteria from high sulphide marine habitats have been found to contain ferrimagnetic greigite, $\mathrm{Fe}_{3} \mathrm{~S}_{4}$, which is isostructural with magnetite [25-28]. An example of a chain of magnetosomes within a bacterial cell is shown in Figure 3.

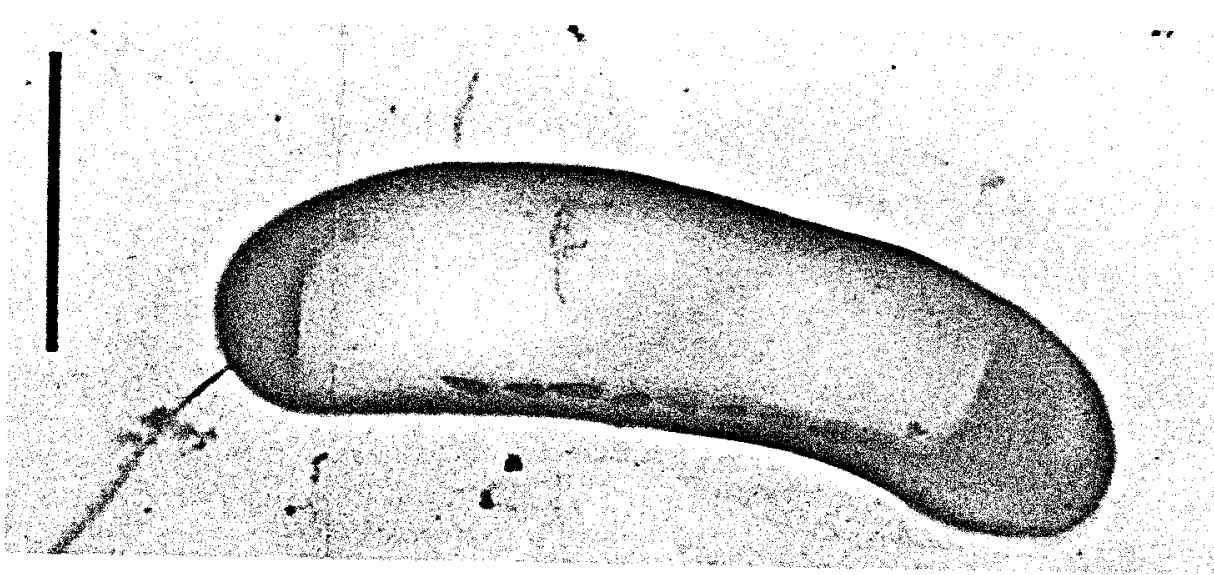

Figure 3. Electron micrograph of a magnetotactic bacterium, showing the chain of magnetosomes, and the flagellum at the left-hand side. The bar is $1 \mu \mathrm{m}$. (Courtesy of N. Blakemore.) 
For a particular type of bacterium, the crystalline magnetosome cores of magnetite have uniform size, shape, crystal morphology and arrangement within the cell. The number of magnetosomes per cell is variable within a population, and depends on the environmental concentration of iron, oxygen, and other chemicals [29]. In most species the magnetosomes occur as a chain of particles arranged along the axis parallel to which the cell moves. The magnetosomes situated at the end of such chains are often smaller than those at the centre. This suggests that the magnetosome chain grows at both ends along the long axis of the cell as iron brought into the cell is mineralised into magnetite. The biomineralisation process, by which the production of the magnetite single crystal is under biological control, is of considerable interest and has been extensively studied [24].

\subsection{Magnetic Properties of the Magnetosome Chain}

The hierarchical structure of the magnetosome chain is significant when one considers its magnetic properties [16]. Firstly, consider the size of the individual particles. Large particles of any magnetic material, including magnetite, can lower their magnetostatic energy by forming magnetic domains and thus reducing the remanent magnetic moment of the particle. Magnetic domains are regions of uniform magnetization which are separated from each other in the particle by transition regions or domain walls. In the domain walls, the direction of magnetization changes smoothly from that of one domain to another. The width of the domain wall is determined by the fundamental magnetic properties of the material, including exchange and anisotropy energies, and is hence a constant for any magnetic material. Thus when the particle dimensions become comparable with the domain wall width, domains cannot form and the particle is forced to remain a single magnetic domain, with uniform and maximum magnetization. For magnetite, this is $92 \mathrm{emu} / \mathrm{g}$. Calculations give $76 \mathrm{~nm}$ as the upper limit for the single magnetic domain size range for equidimensional particles of magnetite [30]. Because of shape anisotropy, the single magnetic domain volume increases with axial ratio for non-equidimensional particles. As a rule of thumb, magnetite particles with long dimensions of the order of $120 \mathrm{~nm}$ or less are single magnetic domains.

The thermal stability of the magnetization in single domain particles is determined by particle volume. The magnetization is oriented along an energetically favourable direction in the particle known as an easy magnetic axis, which for magnetite above the so-called Verwey transition at $118 \mathrm{~K}$ is parallel to a [111] direction. There are several equivalent [111] directions in the lattice and thermal energy can spontaneously excite transitions over the energy barriers associated with the magnetic anisotropy energy corresponding to the difference between hard and easy magnetic axes. This behaviour, known as superparamagnetism, results in a time-averaged loss of remanent magnetization in an ensemble of particles. For single magnetic domain particles above a certain volume the transition rate of the magnetization will be negligible and the particles will retain a permanent magnetization. For magnetite at $300 \mathrm{~K}$, particles with dimensions greater than or equal to about $35 \mathrm{~nm}$ will be permanently magnetized. 
Thus magnetite particles with long dimensions between about 35 and $120 \mathrm{~nm}$ are permanent single magnetic domains at ambient temperature. The magnetite particles produced by magnetotactic bacteria are typically within this size range. Thus these bacteria are not only producing magnetic mineral particles, they are producing permanent single magnetic domain sized particles of that mineral.

When the particles are organized into chains, the magnetic interactions between them cause their magnetic dipole moments to orient parallel to each other along the chain direction. The total magnetic dipole moment of the chain is thus the sum of the moments of the individual particles. By organizing the particles into chains, a bacterium is essentially constructing a permanent magnetic dipole which is sufficiently large to orient the cell in the geomagnetic field as it swims at ambient temperature in water. Thus magnetotaxis is essentially a passive process in which the cell simply swims straight ahead and is steered along the magnetic field lines by the field acting on its magnetic dipole moment. The speed of migration in the field direction $v_{\mathrm{B}}$ is then determined by the average projection of the magnetic dipole moment along the field direction,

$v_{\mathrm{B}}=v_{0} L(\mu B / k T)$

where $v_{0}$ is the forward speed of the cell, $L(\mu B / k T)$ is the Langevin function, $\mu$ is the permanent magnetic dipole moment of the cell and $B$ is the local magnetic field. For $\mu B / k T$ greater than 10 , the speed of migration in the field direction $v_{\mathrm{B}}$ is greater than 0.9 times the forward speed of the cell $v_{0}$. The value of $\mu$ found in magnetotactic bacteria corresponds to a value of $\mu B / k T$ of approximately 10 . Because $L(\mu B / k T)$ approaches 1 asymptotically as $\mu B / k T$ increases, increasing $\mu$ beyond the value actually found would only produce a marginal increase in the migration velocity in the geomagnetic field direction. Thus the bacteria produce an adequate magnetic moment for the purpose, but no more than is necessary.

The bulk magnetic properties of bacterial magnetite have been extensively measured and these data also show that the biologically controlled mineralisation produces magnetic particles with well-defined magnetic properties which optimize their magnetic effectiveness [31].

\section{CONCLUSIONS}

The examples of magnetic fine particles in biological systems, discussed above, illustrate the considerable diversity in this area. A knowledge of the magnetic fine particle behaviour of various biological systems can provide valuable insights into more general aspects of fine particle magnetism. It is hoped that awareness of this connection may lead to improved small particle magnetic materials, either produced directly by biological processes, by modifying materials of biological origin, by mimicking biologically produced materials, or by the improved understanding resulting from the study of biological systems. 


\section{ACKNOWLEDGEMENTS}

The authors gratefully acknowledge the financial support of the UK Science and Engineering Research Council and the Royal Society (DPED) and the US Office of Naval Research (RBF).

\section{REFERENCES}

1. G.C. Ford, P.M. Harrison, D.W. Rice, J.M.A. Smith, A. Treffry, J.L. White and J. Yariv, Phil. Trans. Proc. Roy. Soc. Lond., B304, 551-576 (1984).

2. P.M. Harrison, S.C. Andrews, G.C. Ford, J.M.A. Smith, A. Treffry and J.L. White, in Iron Transport in Microbes, Plants and Animals, G. Winkelmann, D. Van der Helm and J.B. Neilands, Eds., (VCH, Weinheim, 1987) 445-475.

3. F.A. Fischbach, D.W. Gregory, P.M. Harrison, T.G. Hoy and J.M. Williams, J. Ultrastruct. Res., 37, 495-503 (1971).

4. D.P.E. Dickson, N.M.K. Reid, S. Mann, V.J. Wade, R.J. Ward and T.J. Peters, Biochim. Biophys. Acta, 957, 81-90 (1988).

5. T.G. St. Pierre, D.H. Jones and D.P.E. Dickson, J. Magn. Magn. Mater, 69, 276-284 (1987).

6. L. Néel, J. Phys. Soc. Japan, 17 Suppl. B1, 676-689 (1962).

7. W.F. Brown Jr., Phys. Rev., 130, 1677-1685 (1963).

8. N.M.K. Reid, D.P.E. Dickson and D.H. Jones, Hyperfine Interactions, 56, 1487-1490 (1990).

9. T.G. St. Pierre, R.K. Pollard, D.P.E. Dickson, R.J. Ward and T.J. Peters, Biochim. Biophys. Acta, 952, 158-163 (1988).

10. C. Selden, M. Owen, J.M.P. Hopkins and T.J. Peters, Brit. J. Haematol,, 44, 593-603 (1980).

11. R.J. Ward, T.Iancu, J.F. Kirkwood, G.M. Henderson and T.J. Peters, Avian Pathol., 17, 451-464 (1988).

12. D.P.E. Dickson, R.K. Pollard, B. Borch-Iohnsen, R.J. Ward and T.J. Peters, Hyperfine Interactions, 40, 889-892 (1988).

13. S.Mann, V.J. Wade, D.P.E. Dickson, N.M.K. Reid, R.J. Ward and M. O'Connell and T.J. Peters, FEBS Lett., 234, 69-72 (1988).

14. R.B. Blakemore, Science, 190, 377-379 (1975).

15. R.P. Blakemore, Ann. Rev. Microbiol. 36, 217-238 (1982).

16. R.B. Frankel, Ann. Rev. Biophys. Bioeng., 13, 85-103 (1984). 
17. R.B. Frankel and R.P. Blakemore, Bioelectromagnetics, 10, 223-237 (1989).

18. R.P. Blakemore and R.B. Frankel, Sci. Amer., 254, 58-65 (1981).

19. R.P. Blakemore, R.B. Frankel and A.J. Kalmijn, Nature, 286, 384-385 (1980).

20. R.B. Frankel, R.P. Blakemore, F.F. Torres de Araujo, D.M.S. Esquivel and J. Danon, Science, 212, 1269-1270 (1981).

21. F.F. Torres de Araujo, N.A. Germano, L.L. Goncalves, M.A. Pires and R.B. Frankel, Biophys. J., 58, 549-556 (1990).

22. D.A. Bazylinski, R.B. Frankel and H.W. Jannasch, Nature, 333, 518-519 (1988).

23. R.P. Blakemore, N.A. Blakemore, D.A. Bazylinski and T.T. Moench, in Bergey's Manual of Systematic Bacteriology, Vol.3 M.P. Bryant, N. Pfennig and H.T. Staley, Eds., (Williams and Wilkins, Baltimore, 1987).

24. S. Mann and R.B. Frankel, in Biomineralization: Chemical and Biochemical Perspectives, S. Mann, J. Webb and R.J.P. Williams, Eds., (VCH, Weinheim, 1989) 388-426.

25. S. Mann, N.H.C. Sparks, R.B. Frankel, D.A. Bazylinski and H.W. Jannasch, Nature, 343, 258-260 (1990).

26. D.A. Bazylinski, R.B. Frankel, A.J. Garratt-Reed and S. Mann, in Iron Biominerals, R.B. Frankel and R.P. Blakemore, Eds., (Plenum, New York, 1991), 239-255.

27. B.R. Heywood, D.A. Bazylinski, A. Garratt-Reed, S. Mann and R.B. Frankel, Naturwissenschaften, 77, 536-538 (1990).

28. M. Alpert, P. Calvert, R. Frankel, P. Rieke and D. Tirrell, Eds., Materials Syntheses Based on Biological Processes (Materials Research Society, Pittsburgh, 1991) 93-108.

29. R.P. Blakemore, K.A. Short, D.A. Bazylinski, C. Rosenblatt and R.B. Frankel, Geomicrobiol. J., 4 53-71 (1985).

30. R.F. Butler and S.K. Banerjee, J. Geophys. Res., 80, 4049-4058 (1975).

31. B.M. Moskowitz, R.B. Frankel, P.J. Flanders, R.P. Blakemore and B.B. Schwartz, J. Magn. Magn. Mater., 73, 273-288 (1988). 